\title{
Homogeneous antibody-drug conjugates via site-selective disulfide bridging
}

\author{
Nafsika Forte, Vijay Chudasama, and James R. Baker* \\ Department of Chemistry, University College London, London, UK; email: j.r.baker@ucl.ac.uk
}

Antibody-drug conjugates (ADCs) constructed using site-selective labelling methodologies are likely to dominate the next generation of these targeted therapeutics. To this end, disulfide bridging has emerged as a leading strategy as it allows the production of highly homogeneous ADCs without the need for antibody engineering. It consists of targeting reduced interchain disulfide bonds with reagents which reconnect the resultant pairs of cysteine residues, whilst simultaneously attaching drugs. The 3 main reagent classes which have been exemplified for the construction of ADCs by disulfide bridging will be discussed in this review; bissulfones, next generation maleimides and pyridazinediones, along with others in development.

\section{Introduction}

In the past decades, the potential of monoclonal antibodies (mAbs) in targeted therapy against cancer has been realized, with the market of therapeutic antibodies currently being the fastest growing sector in the pharmaceutical industry. While unmodified antibodies have demonstrated unprecedented activities in the clinic, their action can also be synergistically improved by arming them with cytotoxic drugs to create a new class of targeted therapy, i.e. antibody-drug conjugates (ADCs). ${ }^{1,2}$ This strategy has great potential to dramatically reduce the side-effects observed with standard chemotherapy, since the binding specificity of the antibody would facilitate selective drug accumulation at the site of disease, minimizing exposure to healthy tissues. In addition, this can enable the use of ultrapotent payloads, capable of killing cells at sub-nanomolar concentrations, which are too toxic to be used alone in conventional chemotherapy. ${ }^{3}$

To date, out of more than 90 ADCs that have entered clinical trials, only 4 have been approved for clinical use; gemtuzumab ozogamicin (Mylotarg ${ }^{\mathrm{TM}}$ ), ado-trastuzumab emtansine (Kadcyla ${ }^{\mathrm{TM}}$ ), 
inotuzumab ozogamicin (Besponsa ${ }^{\mathrm{TM}}$ ) and brentuximab vedotin (Adcetris ${ }^{\mathrm{TM}}$ ). ${ }^{4}$ The construction of ADCs by attaching the drugs to surface accessible lysine residues (as in Mylotarg ${ }^{\mathrm{TM}}$, Kadcyla $^{\mathrm{TM}}$, Besponsa $^{\mathrm{TM}}$ ) has been heavily pursued due to the limited antibody processing steps required, as well as the formation of a biologically robust amide bond (i.e. via NHS ester chemistry). ${ }^{5}$ However, with over 80 lysine residues present in a typical IgG1 antibody, it has been estimated that this strategy can afford up to $10^{6}$ distinct molecular species when targeting average drug to antibody ratios (DARs) of 2-4. ${ }^{6}$ Furthermore a statistical mixture of DARs is obtained (0-8 drugs per antibody) which has major pharmacokinetic implications. ${ }^{7,8}$

Conjugation of payloads to the cysteine residues generated upon reduction of the four interchain disulfide bonds present in IgG1 can significantly reduce heterogeneity compared to lysine modification. Initial studies had shown that even though reacting all eight sulfhydryl groups can afford homogeneity (DAR 8), the resultant ADC was inferior in vivo, due to high plasma clearance rates and propensity to aggregate. ${ }^{9,10}$ The therapeutic index was shown to significantly increase by reducing the drug load from eight to four, which was achieved by partial reduction and subsequent conjugation of the four disulfide bonds to afford an average DAR of 4 , which led to the development of Adcetris ${ }^{\mathrm{TM}} .8$ Nevertheless, this ADC was shown to have a reduced half-life in vivo due to its formulation still containing a heterogeneous mixture of products with DARs of $0,2,4$, 6 and 8 as the major components. In addition, the use of classical maleimides as the favoured approach for cysteine bioconjugation has been demonstrated to suffer a further limitation; the resultant conjugates are not stable in vivo, due to retro-Michael reactions that result in premature release of the payload, which is then captured by blood thiols. ${ }^{11}$

Overall, the development of next-generation ADCs has been focused on exploring technologies to producing defined conjugates, with pre-clinical data suggesting that homogeneous ADCs lead to improved pharmacokinetic profiles and are likely to dominate future clinical trials. ${ }^{12}$ Methods being explored include the use of recombinant antibody engineering to incorporate cysteine mutants, unnatural amino acids or enzymatic recognition sequences as handles for site-specific conjugation and controlled drug loading. ${ }^{13-15}$ However, these approaches limit global applicability towards all antibody systems, while they add an extra layer of complexity to the ADC production which can increase the manufacturing cost. 
To avoid the need for genetic engineering, the functional rebridging of disulfide bonds has emerged as a leading strategy in recent years for the generation of homogeneous ADCs. By covalently re-connecting the cysteine residues, a controlled loading of one drug per disulfide bond and hence a DAR of 4 in IgG1s can be achieved, while maintaining the stabilizing effect of the disulfide bridge. The modification is distal from the antigen binding or Fc regions, hence even if the insertion of a carbon bridge leads to a minor perturbation in the conformation of the local amino-acid environment the biological activity of the antibody is not compromised. This review is intended to cover recent developments in the area of disulfide bridging for the generation of ADCs.

\section{Reagents employed for disulfide bridging}

\section{Bissulfones}

The concept of disulfide bridging using bissulfone reagents was firstly introduced in the 1990s by Lawton et al. ${ }^{16}$ However, it was not until 2006 that the method gained widespread attention when Brocchini and coworkers reported the application of optimized bissulfone reagents for the PEGylation of therapeutic proteins with the view to extending their in vivo half-life. ${ }^{17,18}$ In the mechanistic pathway, the reactive species is a monosulfone reagent, which is generated in situ upon elimination of $p$-toluene sulfonic acid. Following disulfide bond reduction (usually with DTT or TCEP reducing agents), the two thiols react in sequence with this enone-sulfonyl reagent via an addition-elimination step, followed by a second addition reaction. This eventually results in the two cysteines being re-connected via a three-carbon bridge, with the formation of two diastereomers due to the introduction of a new stereogenic centre. ${ }^{19}$ In peptide studies it has been shown by Weil and coworkers that the rebridging does not always proceed with monosulfones bearing sterically hindered functionalities (e.g. a drug). ${ }^{20}$ However, the incorporation of PEG spacers between the reagent and the functionality has been shown to overcome this limitation whilst also increasing the water solubility of the otherwise insoluble bissulfones. ${ }^{21,22}$ In 2014 , Godwin and coworkers employed a bissulfone reagent attached to monomethyl auristatin $\mathrm{E}$ (MMAE), through a PEG 24 spacer with an enzymatically cleavable val-cit-PABC linker, to rebridge the disulfide bonds of trastuzumab (Scheme 1a). ${ }^{21}$ This strategy successfully afforded an ADC that contained the DAR 4 species as the major product (78\%). ELISA analysis confirmed 
that binding to HER2 antigen was maintained, and the ADC was shown to be stable upon incubation in $50 \%$ rat or undiluted human serum for $96 \mathrm{~h}$ at $37{ }^{\circ} \mathrm{C}$. In addition potent antiproliferative activity was demonstrated against antigen positive cell lines in vitro, and in vivo the disulfide-bridged ADC exhibited improved potency compared to unconjugated trastuzumab, although multiple high doses (e.g. 5 doses of $20 \mathrm{mg} / \mathrm{kg}$ ) were required for tumour growth inhibition. Furthermore, by comparing the relative efficacy of different DAR variants $(1,2,3$, and 4) isolated by hydrophobic interaction chromatography (HIC) purification, a clear dose-response was obtained based on drug loading. Only the DAR 4 species exhibited 100\% tumour growth inhibition, when three doses of $20 \mathrm{mg} / \mathrm{kg}$ were administered. ${ }^{23}$

A later report published by Pabst and coworkers in 2017 demonstrated that the need for multiple high doses previously required for tumour growth inhibition was likely due to loss of the MMAE payload in circulation, prior to reaching the tumour. ${ }^{24}$ This was revealed to be due to enzymatic cleavage of the valine-citrulline-PABC spacer by serum proteases and not due to chemical instability of the linker. ${ }^{25}$ Through reagent optimization, it was observed that by shielding the valine-citrulline-PABC-MMAE moiety with a branched PEG 24 linker, more stable constructs can be produced. By using this approach, two novel ADCs were generated with DARs of 4 and 8 , respectively; the latter was synthesized by employing a bissulfone moiety that contained two molecules of MMAE and two branched PEG 24 arms (Scheme 1b and 1c, respectively). Both ADCs were shown to be more efficacious than Adcetris ${ }^{\mathrm{TM}}$, while an increase in potency from 4 to 8 drugs per antibody was observed, with complete tumour growth regression at a single dose of 1 $\mathrm{mg} / \mathrm{kg}$ and $0.5 \mathrm{mg} / \mathrm{kg}$, respectively. These results demonstrate that the drug-linker design is a critical parameter in enhancing the ADC potency in vivo.

The characterization of these bissulfone ADCs in the literature has been largely limited to the use of HIC and size-exclusion chromatography, and the absence of SDS-PAGE or detailed mass spectrometry characterization should be noted. These methods are required to reveal a more thorough understanding of the outcome of the conjugation reactions, including the interchain connectivity pattern of the rebridged disulfide bonds and the homogeneity of the conjugates. For example, given the outcome with other bridging reagents described below, it should be assumed that these bissulfone protocols give a mixture of disulfide connectivity in the hinge region. 


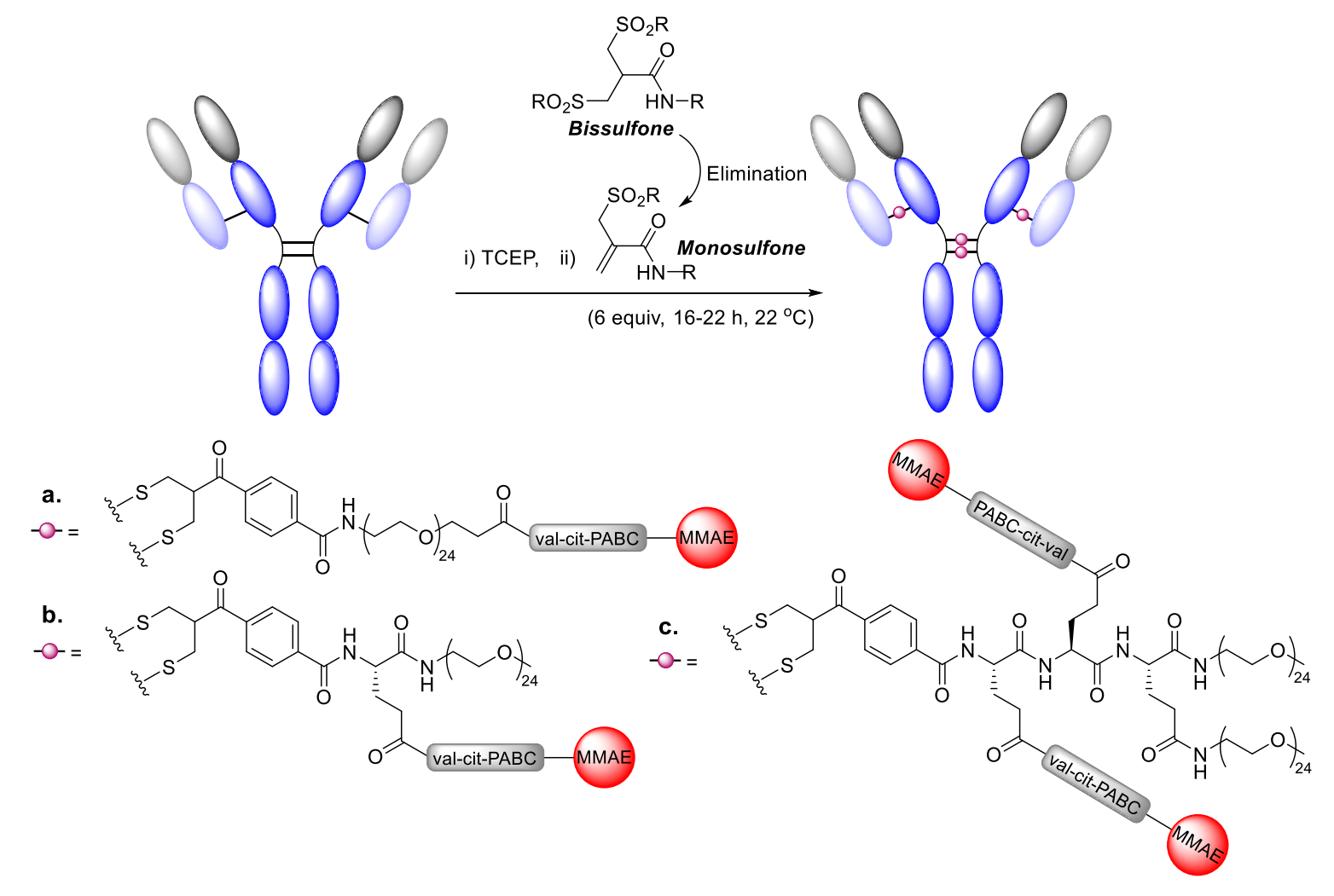

Scheme 1 - ADC construction by disulfide bridging with bissulfone reagents: a. bissulfone-PEG 24-valcit-PABC-MMAE reagent, b. bissulfone reagent of increased stability, consisting of a PEG 24 chain and val-cit-PABC-MMAE attached via a glutamic acid moiety, c. bissulfone reagent used to construct a DAR

8 ADC, consisting of 2 val-cit-PABC-MMAE moieties, and 2 PEG 24 chains; black bars on antibody denote disulfide bonds.

\section{Next generation maleimides (NGMs)}

In 2010, Baker, Caddick and coworkers reported on the next generation maleimides (NGMs) as highly efficient cysteine cross-linking reagents. ${ }^{26}$ The incorporation of two leaving groups (e.g. bromine or thiophenol) across the double bond of the widely used maleimide motif enables two consecutive addition-elimination sequences to take place upon reaction with a reduced disulfide bond to reconnect it with a rigid two-carbon bridge. Since their conception, NGMs have been successfully employed in disulfide bridging and their use has significantly expanded to a variety of applications, including reactive handles in polymers, ${ }^{27}$ protein PEGylation, ${ }^{28}$ antibody-fragment diagnostics ${ }^{29}$ nuclear imaging ${ }^{30}$ photochemistry ${ }^{31}$ and photodynamic therapy ${ }^{32}$. However, their 
greatest potential has been realized in the formation of homogeneous ADCs with controlled drug loadings.

In an early example, a dithiophenolmaleimide with an alkyne handle was employed to rebridge the disulfide bonds of trastuzumab, which were then functionalized with doxorubicin via click chemistry (Scheme 1a). ${ }^{33}$ Through a screen of reduction/ conjugation conditions, it was observed that TCEP-based reduction protocols gave DARs between 3 and 4, while methods employing benzeneselenol yielded DARs between 1 and 2. The use of benzeneselenol however has not be pursued further in this role due to its unfavourable poor water solubility and malodour, and thus TCEP has been the reducing agent of choice in future applications. SDS-PAGE and LCMS analysis of these NGM-ADCs revealed the presence of two isomeric products. The first represented the native bridging connectivity, and the second contained an intrachain bridge between the hinge cysteines. This competition between regioisomers occurs due to the proximity of the two cysteines in the heavy chain, which are separated by just two proline residues. Nevertheless, the two antibody chains are held together in vivo by strong non-covalent interactions and recent work by Lyon et al. has demonstrated that the absence of covalent bonds between the heavy chains does not impact the pharmacological properties of the ADC. ${ }^{34}$

The NGM platform was then used to create an industry relevant ADC by employing a dithiophenolmaleimide attached to MMAE through a non-cleavable PEG 12 spacer (Scheme $1 \mathrm{~b}$ ). ${ }^{35}$ Upon incubation of the initially generated dithiomaleimide in human serum, thiol exchange with albumin was shown to occur within four days, in a comparable rate to that observed with classical maleimide conjugates, though via a different mechanism. By quantitative hydrolysis of the maleimide moieties, robust serum stability was observed. The resultant ADC had an average DAR of 3.89 and showed potent antiproliferative activity in vitro, as well as in vivo, where complete tumour regression was achieved after three $20 \mathrm{mg} / \mathrm{kg}$ doses. ${ }^{36}$ Interestingly, high potency was observed despite the use of a non-cleavable linker, in contrast to previous data suggesting the use of a cleavable linker is essential for release of free MMAE to induce cytotoxic activity. ${ }^{37}$

While post-conjugation hydrolysis was key to ensure serum stability, the conditions initially employed were sub-optimal, since they required incubation in $\mathrm{pH} 8.4$ buffer for $72 \mathrm{~h}$. In a follow up report, it was demonstrated that dibromomaleimides with electron withdrawing C-2 (glycine derived) linkers offer an optimized protocol for the use of NGMs to construct of serum stable DAR 
4 ADCs ${ }^{38}$ Conjugation was shown to occur in $<1$ minute, which was followed by quantitative hydrolysis in just over $1 \mathrm{~h}$. Extensive LC-MS characterization revealed that by employing these fast-hydrolyzing linkers, improved homogeneity was also inferred, by preventing undesired sidereactions that could occur upon prolonged incubation in basic $\mathrm{pH}$ buffer. The conjugates showed complete stability in physiological $\mathrm{pH}$, as well as $\mathrm{pH} 5.5$, with the later approximating endosomal $\mathrm{pH}$ and hence the most acidic conditions an antibody is likely to experience prior to lysosomal degradation. Interestingly cleavage of the imide bond to release the amine and form the corresponding anhydride was shown to occur upon prolonged incubation of maleamic acids in lysosomal $\mathrm{pH}$ (i.e. 4.5 , for $72 \mathrm{~h}, 37^{\circ} \mathrm{C}$ ), a feature which was used with a self-immolative PABC linker to represent a prospective new mechanism for lysosomal drug release. ${ }^{39}$

Additionally, in a study published in 2015 by Jackson and coworkers, DAR 4 ADCs were prepared using a dibromomaleimide attached to monomethyl auristatin F (MMAF) via a non-cleavable linker and were directly compared to analogous heterogeneous ADCs generated by stochastic labelling using classical maleimide versions. ${ }^{40}$ All constructs contained identical linkers and payloads, with the view to determining the relative contributions of homogeneity and disulfide bridging in ADC production. The NGM ADC showed complete tumour remission when two 3 $\mathrm{mg} / \mathrm{kg}$ doses were administered. Furthermore in NOG mice, which are insensitive to trastuzumab thus allowing a clearer picture of the influence of conjugation strategy, the NGM-ADC led to a significant delay in tumor growth whilst the classical maleimide-ADC led to no significant inhibition relative to controls. The results provide convincing evidence that NGM ADCs are potent, and that the improved homogeneity infers superior efficacy. It should be noted that the NGM ADCs used in this study were not hydrolyzed post conjugation, and thus a further improvement due to enhanced serum stability could be anticipated. 


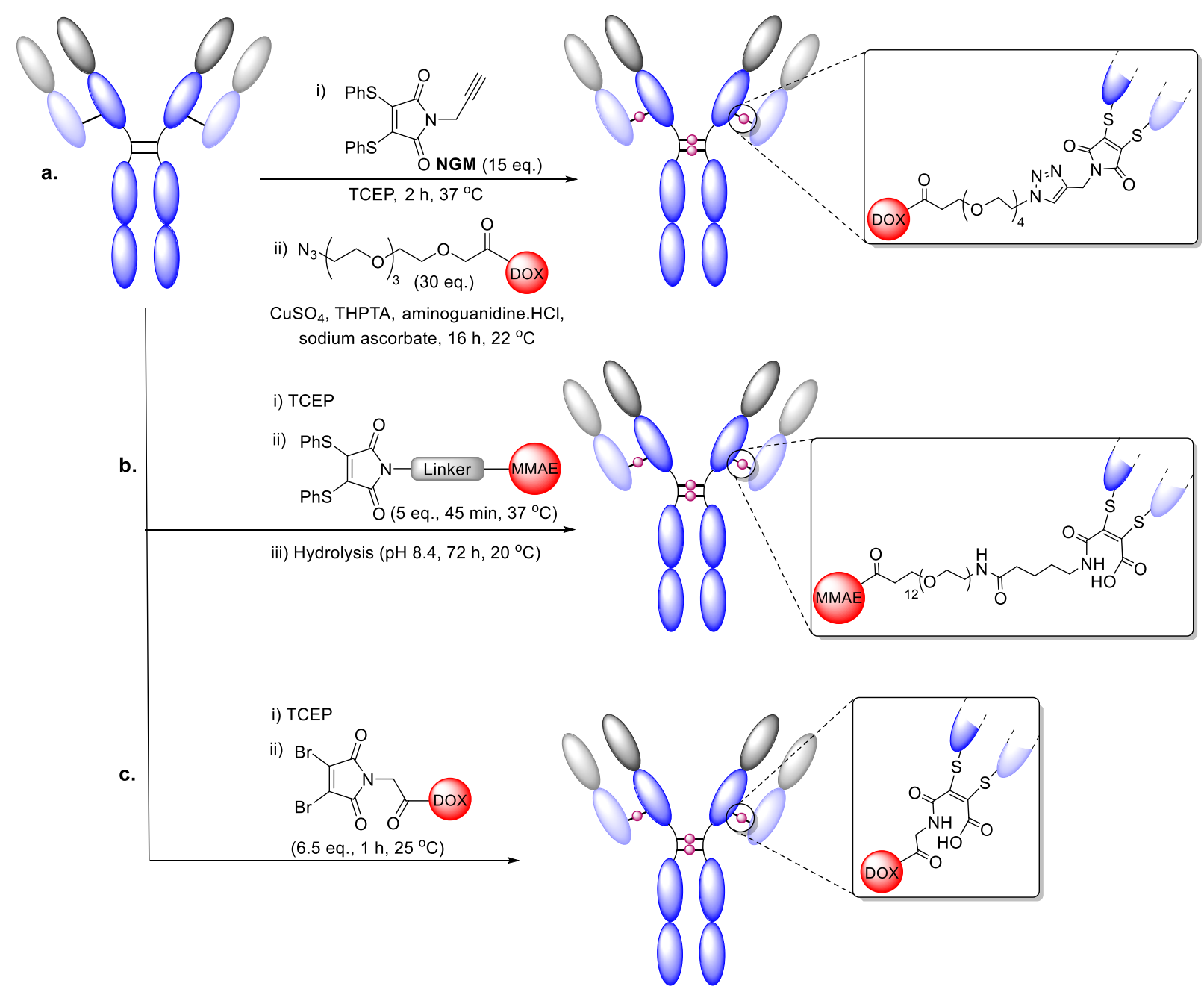

Scheme 2 - ADC construction by disulfide bridging using next-generation maleimides; a. dithiophenolmaleimide with alkyne handle, b. dithiophenolmaleimide attached to MMAE through a non-cleavable C6PEG 12 spacer, c. optimized DAR 4 ADC preparation using a dibromomaleimide containing a C2 spacer, linked to doxorubicin.

\section{Pyridazinediones}

Following on from the successful development of NGMs, Chudasama and Caddick et al. reported on the use of dibromopyridazinediones (PDs) in 2011 as a versatile class of disulfide-bridging reagents that ever since have demonstrated significant potential in the synthesis of homogeneous ADCs. Bridging occurs via a similar manner to NGMs involving two consecutive addition- 
elimination sequences. Although structurally similar to NGMs, PDs contain four points of chemical attachment, a feature that was exploited to develop a platform for the dual-labeling of trastuzumab, which could find important applications in imaging and theranostics (Scheme $3 a) .{ }^{41}$ More specifically, a PD reagent was constructed to contain two orthogonal clickable handles; a linear alkyne and a strained alkyne. Following disulfide bond reduction and bridging, the antibody was functionalized with doxorubicin and sulfo-Cy5 fluorophore via strain-promoted and copper(I)-catalyzed azide-alkyne cycloadditions, respectively. UV/Vis spectroscopy confirmed that each species was attached with a loading of 4 and binding activity was maintained. Interestingly, SDS-PAGE analysis showed retention of the full antibody structure, with minimal amount of half-antibody $(<5 \%)$. In a later publication, it was highlighted that this was due to an in situ bridging protocol being carried out at $4{ }^{\circ} \mathrm{C}$, in which the reducing agent and PD molecule were added in tandem, preventing disulfide bond shuffling, by immediate re-connection of the open disulfides. $^{42}$

Based on the observation that dithiophenol-pyridazinediones do not exhibit any cross-reactivity with TCEP, Chudasama et al. also developed a first-in class reagent that contained TCEPfunctionalities tethered onto the thiophenol groups of the PD scaffold to effect both reduction and rebridging in one step (Scheme 3b). ${ }^{43}$ An alkyne handle was also incorporated into the structure to enable later functionalization via click chemistry. Incubation of this molecule with trastuzumab was shown to afford fully rebridged antibody in an interchain fashion, demonstrating again that immediate re-bridging upon reduction is key to prevent disulfide scrambling. However, it should be noted that this reagent is prone to oxidation upon storage and should be prepared fresh prior to use.

In a follow up report, Chudasama and Baker et al. employed the PD platform to create industry relevant ADCs containing the potent MMAE anti-cancer drug (Scheme 3c). ${ }^{36}$ This was attached to the PD functionality via either a non-cleavable PEG 12 spacer or a cleavable valine-citrulline linker to allow for a direct comparison. Following bridging, HIC analysis demonstrated that $90 \%$ DAR 4 species was obtained, which represents the best control of loading for such reagents in the field. Upon incubation in human serum, minimal transfer to albumin was shown to occur over 7 days at $37^{\circ} \mathrm{C}$. Potent antiproliferative activity was observed in vitro for both ADCs, although the conjugate with the cleavable linker was $c a$. 100x more potent. In vivo efficacy studies were also 
performed for both ADCs, where complete tumour regression was achieved after three doses of $20 \mathrm{mg} / \mathrm{kg}$ were administered, demonstrating again that it is useful, but not essential for the MMAEADCs to have a cleavable linker.

In a recent publication by Wang and coworkers, disulfide-bridged ADCs containing the antitubulin drug DM1 were also synthesized using the PD platform. ${ }^{44}$ The payload was attached on one arm of a dithiophenol-pyridazinedione moiety via a non-cleavable SMCC linker, while PEG spacers of different lengths (6 and 26 units) were incorporated onto the other arm in order to increase the solubility of the hydrophobic DM1. The longer PEG spacer was shown to be most efficient for conjugation, with no organic co-solvent required, minimizing the potential for aggregation. Both ADCs achieved complete tumour regression after two doses of $10 \mathrm{mg} / \mathrm{kg}$ were administered, exhibiting similar efficacy with Kadcyla. However, at lower doses, the construct with the longer PEG spacer was less potent due to the branched polymer hindering DM1microtubule interactions. These results suggest that a cleavable linker should be incorporated into the reagent design in order to achieve maximal efficacy.

Furthermore, while a drug loading of 4 has been demonstrated to afford the best therapeutic index, for certain hydrophobic drugs, such as the highly potent pyrrolobenzodiazepine dimer (PBD), a loading of 2 is preferred, since higher loadings can result in aggregate formation. ${ }^{45}$ So far, this has only been addressed through genetic engineering, e.g. THIOMABs. ${ }^{46}$ Chudasama and coworkers developed a method to address this issue starting from a native antibody construct. ${ }^{47}$ It was demonstrated that a bifunctional dibromopyridazinedione PEG reagent, containing a single alkyne handle, could "tie-up" 2 pairs of the 4 disulfides on trastuzumab to allow formation of a conjugate with an overall loading of 2 modules through click reaction (Scheme $3 \mathrm{~d}$ ). The linker design was also extended to incorporate 2 alkyne handles per bifunctional PD molecule to achieve an overall loading of 3.9, demonstrating the versatility of this approach. 
a.

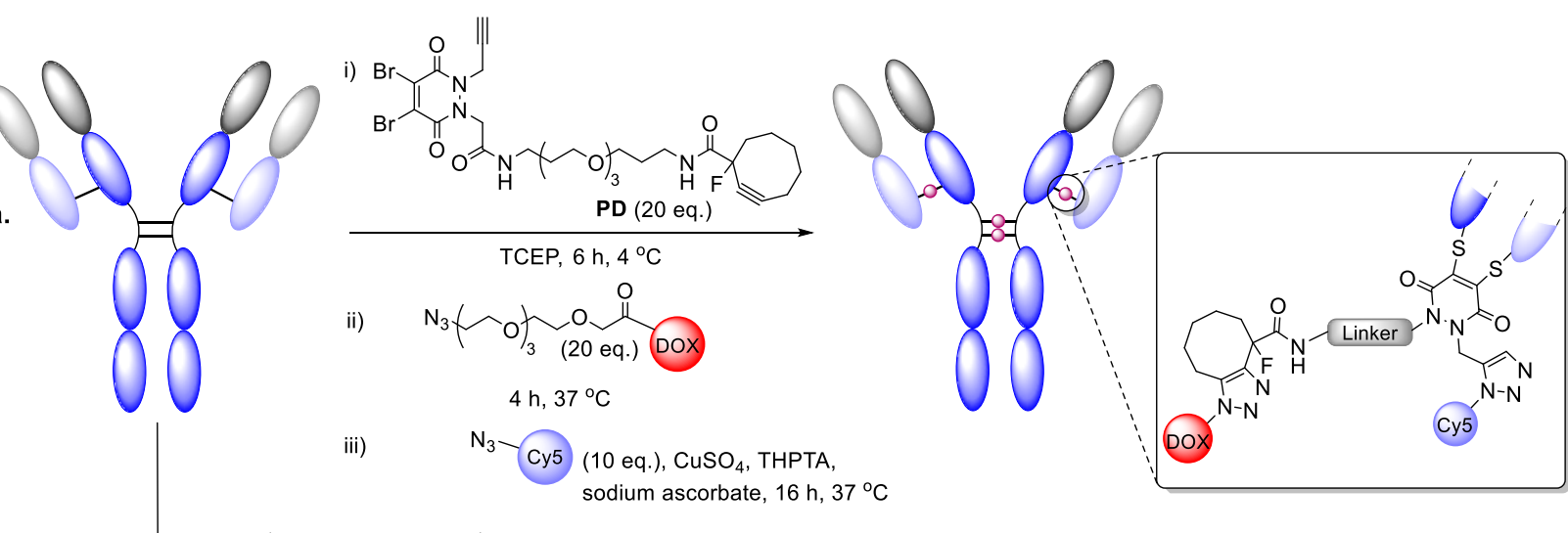

b.

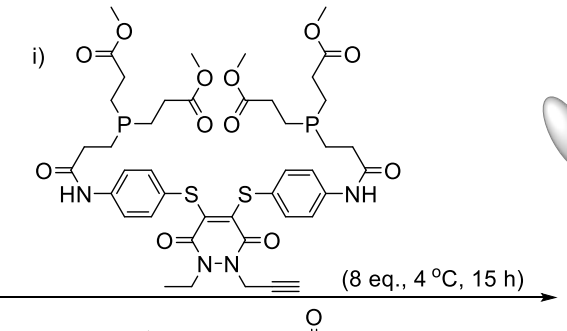

ii)

$\mathrm{N}_{3} \mathrm{O}_{3} \mathrm{~T}_{(20 \mathrm{eq})}^{\mathrm{O}} \mathrm{O}$ (DOX

$$
\mathrm{CuSO}_{4} \text {, THPTA, }
$$

sodium ascorbate, $1.5 \mathrm{~h}, 21^{\circ} \mathrm{C}$

c.

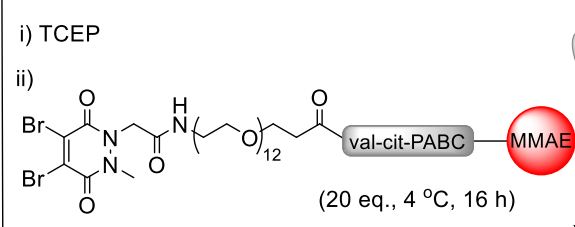

d.

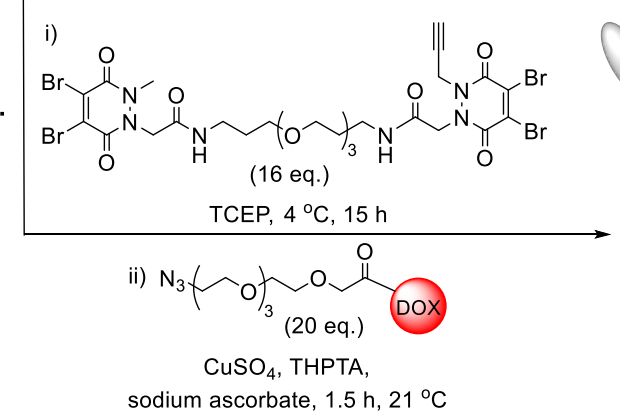

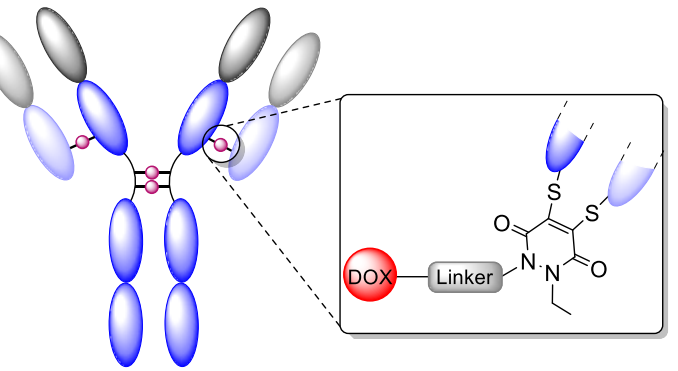

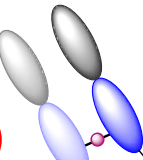

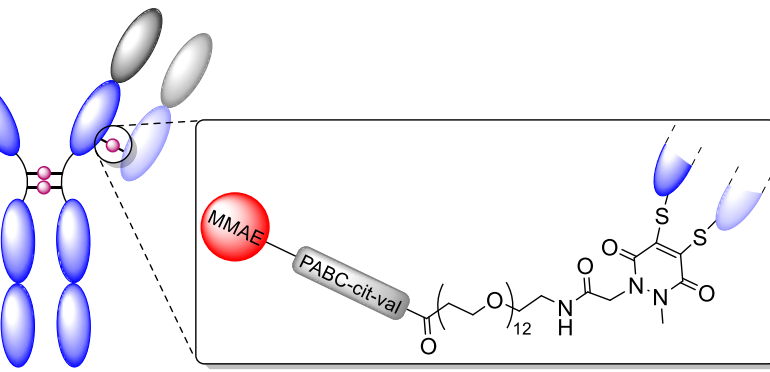

Scheme 3 - ADC construction by disulfide bridging using pyridazinediones; a. dibromopyridazinedione with orthogonal clickable handles, b. dithiophenolpyridazinedione "2-in-1" reagent to effect both reduction and rebridging at a single step, c. dibromopyridazinedione attached to MMAE through a cleavable PEG 12val-cit-PABC spacer (a non-cleavable linker was synthesized in the same way, without the val-cit-PABC spacer), d. bifunctional dibromopyridazinedione reagent employed to create a DAR 2 ADC. 


\section{Other reagents}

The extensive work carried out with bissulfones, next-generation maleimides and pyridazinediones has clearly demonstrated the merits of disulfide rebridging in producing highly homogeneous constructs. In recent years, a few novel reagents have been described for disulfide bridging on antibodies or antibody fragments, though they have not been elaborated yet in the literature; an overview is given in this chapter.

Concortis Biosystems has developed the C-Lock ${ }^{\mathrm{TM}}$ conjugation technology, utilizing dibromomethyl heterocyclic aromatic groups (such as quinoxaline, triazole) to produce disulfide bridged ADCs (Figure 4a). ${ }^{48}$ The resultant constructs are currently in preclinical/ Phase I clinical trials, but no data has been published as of the time of this review. The use of 1,3-dichloroacetone as a simple two-step strategy to create homogeneous ADCs has also been described in a patent application by Novartis AG. ${ }^{49}$ In this approach, an acetone-linked bridge is formed, which is further reacted via oxime ligation to attach the payload (Scheme 4b). While no data on the antibody bridging efficiency and ADC performance have been publicly available yet, various reports in the literature employing this strategy in bridging single-disulfide containing peptides or proteins have demonstrated the applicability of this approach. ${ }^{50,51}$

A novel disulfide bridging strategy utilizing the arylene-dipropionitrile (ADPN) functionality has recently been reported by Wagner et al. (Scheme 4c). ${ }^{52}$ The bridging efficiency of meta, ortho and para isomers of ADPN was shown to differ markedly, as exemplified on trastuzumab. The paraand ortho-isomers were demonstrated to be less efficient bridging reagents, hypothesized to be due to instability to hydrolysis. In contrast, meta-ADPN was shown to be more efficient, with full and half-antibody being the main species observed by SDS-PAGE analysis, along with several other bands indicative of incomplete bridging. By utilizing these meta ADPNs, two ADCs were created incorporating MMAE and a $\beta$-galactosidase-responsive self-immolative linker, with and without a PEG spacer. Native mass spectrometry analysis of the conjugates demonstrated that although the major species corresponds to DAR 4, a significant amount of other DARs (particularly DAR 5) is also present. Such higher loaded species are proposed to be formed when the reduced disulfide reacts with two of the ADPN molecules, in competition with bridging, and may suggest that such longer (5-carbon) bridges reduce the efficiency of the cysteine cross-linking. 
Nevertheless, both ADCs were shown to selectively kill HER2-positive cells in vitro with $\mathrm{IC}_{50}$ values comparable to Kadcyla.

A photochemical disulfide bridging approach through a thiol-yne coupling has also recently been described by Griebenow and coworkers (Scheme 4d) ${ }^{53}$ Disulfide rebridging of the M14-G07 Fab fragment with 6-heptynoic acid was achieved upon irradiation with UV light $(365 \mathrm{~nm})$, in the presence of a radical initiator. While no functionality was attached, the presence of a carboxylic acid offers a potential point of attachment. However, despite the novelty of this strategy, bridging was shown to occur only at very high antibody concentrations (i.e. $1 \mathrm{mM}$ ), where a low conjugation yield of $40 \%$ was still obtained.

Notably, apart from imparting functionality with the view to creating antibody conjugates, disulfide bridging has also been employed to enhance the stability and activity of antibody fragments. Bernardes et al. demonstrated that bridging of antibody fragments with 3,3bis(bromomethyl) oxetane can enhance receptor binding, as well as provoke a slight increase in the degree of flexibility (Scheme 4e) ${ }^{54}$ Furthermore, Pentelute and coworkers showed that bridging of a HER2 affibody with hexafluorobenzene can increase the $\alpha$-helical content of the fragment (Scheme 4f). ${ }^{55}$ 


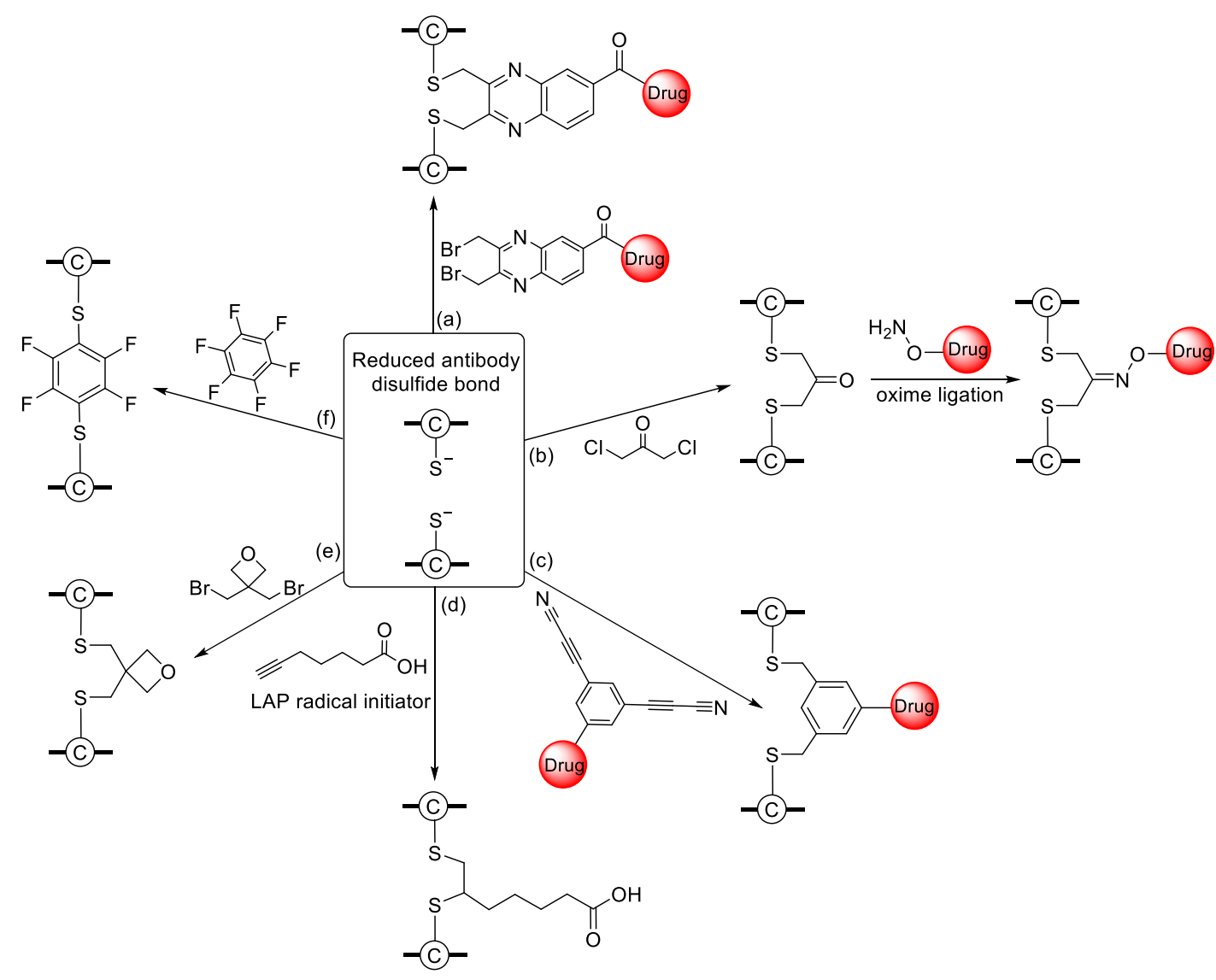

Scheme 4 - Other reagents employed for disulfide bridging on antibodies or antibody fragments; a. CLock $^{\mathrm{TM}}$ technology employing dibromomethyl heterocyclic aromatic groups, b. dichloroacetone, followed by oxime ligation, c. arylene-dipropionitrile (ADPN), d. thiol-yne coupling with 6-heptynoic acid, e. 3,3bis(bromomethyl) oxetane, f. hexafluorobenzene.

\section{Conclusion}

Since its first inception, disulfide rebridging has gained increasing momentum for the development of homogeneous ADCs. This strategy has demonstrated significant advantages over existing techniques, including controlled drug loading, elimination of high DAR species that lead to aggregation, whilst avoiding the need for genetic engineering and being directly applicable on native antibody constructs. So far, three leading disulfide bridging reagents have been reported and characterized extensively in the literature. Bissulfones were the first reagents to be developed for this purpose and paved the way for a new generation of antibody constructs. Various in vivo studies were performed that contributed in understanding the effect that DAR has on tumour 
growth inhibition, as well the influence of drug-linker design in ADC stability and potency. Nextgeneration maleimides represent the fastest disulfide bridging reagents reported to date, with conjugation occurring in $<1$ minute and robustly stable ADCs are generated in just over $1 \mathrm{~h}$ upon subsequent in situ hydrolysis. In addition, the NGM cross-linking strategy was the first to be directly compared to analogous heterogeneous ADCs, with in vivo data demonstrating that superior efficacy is obtained by disulfide-bridging. Pyridazinediones were shown to offer the best control of DAR loading in the field, with 90\% DAR 4 species being obtained. Extensive reagent development was also carried out that demonstrated the versatility of these reagents in synthesizing dually-labeled or DAR 2 ADCs, and an all-in-one reduction and bridging strategy was exemplified.

Various alternative disulfide bridging reagents have been developed in recent years with distinct reactivity profiles, but which have been applied predominantly to peptides, e.g. for stapling. ${ }^{56} \mathrm{~A}$ few of them have been employed in bridging antibody disulfide bonds, though insufficient literature characterization at the moment prevents a direct comparison with previously established reagents.

The disulfide bridging field is growing at a very fast pace, with pre-clinical data suggesting that ADCs of improved pharmacological properties are obtained through this strategy. Given the ease of the approach, and its applicability to native antibodies 'off-the-shelf', it is expected that disulfide-bridged conjugates will play an important role in the development of the next generation of these promising targeted therapeutics.

\section{References}

1 A. M. Wu and P. D. Senter, Arming antibodies: prospects and challenges for immunoconjugates., Nat. Biotechnol., 2005, 23, 1137-1146.

2 J. R. Adair, P. W. Howard, J. A. Hartley, D. G. Williams and K. A. Chester, Antibodydrug conjugates - a perfect synergy, Expert Opin. Biol. Ther., 2012, 12, 1191-1206.

3 G. Casi and D. Neri, Antibody-drug conjugates and small molecule-drug conjugates: opportunities and challenges for the development of selective anticancer cytotoxic agents, J. Med. Chem., 2015, 58, 8751-5761. 
4 T. Rodrigues and G. J. L. Bernardes, Development of antibody-directed therapies: Quo Vadis?, Angew. Chem. Int. Ed., 2018, 57, 2032-2034.

5 M. Gordon, M. Canakci, L. Li, J. Zhuang, B. A. Osborne and S. Thayumanavan, A field guide to challenges and opportunities in antibody-drug conjugates for chemists., Bioconjugate Chem., 2015, 26, 2198-2215.

6 L. Wang, G. Amphlett, W. A. Blattler, J. M. Lambert and W. Zhang, Structural characterization of the maytansinoid-monoclonal antibody immunoconjugate, huN901DM1, by mass spectrometry, Protein Sci., 2005, 14, 2436-2446.

7 I. E. Krop, M. Beeram, S. Modi, S. F. Jones, S. N. Holden, W. Yu, S. Girish, J. Tibbitts, J. H. Yi, M. X. Sliwkowski, F. Jacobson, S. G. Lutzker and H. A. Burris, Phase I study of trastuzumab-DM1, an HER2 antibody-drug conjugate, given every 3 weeks to patients with HER2-positive metastatic breast cancer, J. Clin. Oncol., 2010, 28, 2698-2704.

8 K. J. Hamblett, P. D. Senter, D. F. Chace, M. M. C. Sun, J. Lenox, C. G. Cerveny, K. M. Kissler, S. X. Bernhardt, A. K. Kopcha, R. F. Zabinski, D. L. Meyer and J. A. Francisco, Effects of drug loading on the antitumor activity of a monoclonal antibody drug conjugate, Clin. Cancer Res., 2004, 10, 7063-7070.

9 S. O. Doronina, B. E. Toki, M. Y. Torgov, B. A. Mendelsohn, C. G. Cerveny, D. F. Chace, R. L. Deblanc, R. P. Gearing, T. D. Bovee, C. B. Siegall, J. A. Francisco, A. F. Wahl, D. L. Meyer and P. D. Senter, Development of potent monoclonal antibody auristatin conjugates for cancer therapy, Nat. Biotechnol., 2003, 21, 778-785.

10 N. S. Beckley, K. P. Lazzareschi, H. Chih, V. K. Sharma and H. L. Flores, Investigation into temperature-induced aggregation of an antibody drug conjugate, Bioconjugate Chem., 2013, 24, 1674-1683.

11 P. A. Szijj, C. Bahou and V. Chudasama, Minireview: Addressing the retro-Michael instability of maleimide bioconjugates, Drug Discov. Today Technol., 2018, xxx, 1-8.

12 D. Y. Jackson, Processes for constructing homogeneous antibody drug conjugates, Org. Process Res. Dev., 2016, 20, 852-866.

13 J. R. Junutula, H. Raab, S. Clark, S. Bhakta, D. D. Leipold, S. Weir, Y. Chen, M. 
Simpson, S. P. Tsai, M. S. Dennis, L. Yanmei, Y. G. Meng, C. Ng, J. Yang, C. C. Lee, E.

Duenas, J. Gorrell, V. Katta, A. Kim, K. McDorman, K. Flagella, R. Venook, S. Ross, S.

D. Spencer, W. Lee Wong, H. B. Lowman, R. Vandlen, M. X. Sliwkowski, R. H. Scheller, P. Polakis and W. Mallet, Site-specific conjugation of a cytotoxic drug to an antibody improves the therapeutic index, Nat. Biotechnol., 2008, 26, 925-932.

14 S. A. Kularatne, V. Deshmukh, J. Ma, V. Tardif, R. K. V Lim, H. M. Pugh, Y. Sun, A. Manibusan, A. J. Sellers, R. S. Barnett, S. Srinagesh, J. S. Forsyth, W. Hassenpflug, F. Tian, T. Javahishvili, B. Felding-Habermann, B. R. Lawson, S. A. Kazane and P. G. Schultz, A CXCR4-targeted site-specific antibody-drug conjugate., Angew. Chem. Int. Ed., 2014, 53, 11863-11867.

15 P. M. Drake, A. E. Albers, J. Baker, S. Banas, R. M. Barfield, A. S. Bhat, G. W. de Hart, A. W. Garofalo, P. Holder, L. C. Jones, R. Kudirka, J. McFarland, W. Zmolek and D. Rabuka, Aldehyde tag coupled with HIPS chemistry enables the production of ADCs conjugated site-specifically to different antibody regions with distinct in vivo efficacy and PK outcomes., Bioconjugate Chem., 2014, 25, 1331-41.

16 F. A. Liberatore, R. D. Comeau, J. M. McKearin, D. A. Pearson, B. Q. Belonga, S. J. Brocchini, J. Kath, T. Phillips, K. Oswell and R. G. Lawton, Site-directed chemical modification and cross-linking of a monoclonal antibody using equilibrium transfer alkylating cross-link reagents, Bioconjugate Chem., 1990, 1, 36-50.

17 S. Shaunak, A. Godwin, J.-W. Choi, S. Balan, E. Pedone, D. Vijayarangam, S. Heidelberger, I. Teo, M. Zloh and S. Brocchini, Site-specific PEGylation of native disulfide bonds in therapeutic proteins., Nat. Chem. Biol., 2006, 2, 312-313.

18 S. Balan, J.-W. Choi, A. Godwin, I. Teo, C. M. Laborde, S. Heidelberger, M. Zloh, S. Shaunak and S. Brocchini, Site-specific PEGylation of protein disulfide bonds using a three-carbon bridge., Bioconjugate Chem., 2007, 18, 61-76.

19 A. Pfisterer, K. Eisele, X. Chen, M. Wagner, K. Müllen and T. Weil, Bioactive unnatural somatostatin analogues through bioorthogonal iodo-and ethynyl-disulfide intercalators, Chem. Eur. J., 2011, 17, 9697-9707. 
20 T. Wang, Y. Wu, L. Kuan, O. Dumele, M. Lamla, D. Y. W. Ng, M. Arzt, J. Thomas, J. O. Mueller, C. Barner-Kowollik and T. Weil, A disulfide intercalator toolbox for the sitedirected modification of polypeptides, Chem. Eur. J., 2015, 21, 228-238.

21 G. Badescu, P. Bryant, M. Bird, K. Henseleit, J. Swierkosz, V. Parekh, R. Tommasi, E. Pawlisz, K. Jurlewicz, M. Farys, N. Camper, X. Sheng, M. Fisher, R. Grygorash, A. Kyle, A. Abhilash, M. Frigerio, J. Edwards and A. Godwin, Bridging disulfides for stable and defined antibody drug conjugates., Bioconjugate Chem., 2014, 25, 1124-1136.

22 S. Brocchini, A. Godwin, S. Balan, J. Choi, M. Zloh and S. Shaunak, Disulfide bridge based PEGylation of proteins., Adv. Drug Deliv. Rev., 2008, 60, 3-12.

23 P. Bryant, M. Pabst, G. Badescu, M. Bird, W. McDowell, E. Jamieson, J. Swierkosz, K. Jurlewicz, R. Tommasi, K. Henseleit, X. Sheng, N. Camper, A. Manin, K. Kozakowska, K. Peciak, E. Laurine, R. Grygorash, A. Kyle, D. Morris, V. Parekh, A. Abhilash, J. W. Choi, J. Edwards, M. Frigerio, M. P. Baker and A. Godwin, In vitro and in vivo evaluation of cysteine rebridged trastuzumab-MMAE antibody drug conjugates with defined drug-toantibody ratios, Mol. Pharm., 2015, 12, 1872-1879.

24 M. Pabst, W. McDowell, A. Manin, A. Kyle, N. Camper, E. De Juan, V. Parekh, F. Rudge, H. Makwana, T. Kantner, H. Parekh, A. Michelet, X. B. Sheng, G. Popa, C. Tucker, F. Khayrzad, D. Pollard, K. Kozakowska, R. Resende, A. Jenkins, F. Simoes, D. Morris, P. Williams, G. Badescu, M. P. Baker, M. Bird, M. Frigerio and A. Godwin, Modulation of drug-linker design to enhance in vivo potency of homogeneous antibodydrug conjugates, J. Control. Release, 2017, 253, 160-164.

25 M. Dorywalska, R. Dushin, L. Moine, S. E. Farias, D. Zhou, T. Navaratnam, V. Lui, A. Hasa-Moreno, M. G. Casas, T.-T. Tran, K. Delaria, S.-H. Liu, D. Foletti, C. J. O’Donnell, J. Pons, D. L. Shelton, A. Rajpal and P. Strop, Molecular basis of valine-citrulline-PABC linker instability in site-specific ADCs and its mitigation by linker design, Mol. Cancer Ther., 2016, 15, 958-970.

26 M. E. B. Smith, F. F. Schumacher, C. P. Ryan, L. M. Tedaldi, D. Papaioannou, G. Waksman, S. Caddick and J. R. Baker, Protein modification, bioconjugation, and disulfide bridging using bromomaleimides, J. Am. Chem. Soc., 2010, 132, 1960-1965. 
27 M. W. Jones, R. A. Strickland, F. F. Schumacher, S. Caddick, J. R. Baker, M. I. Gibson and D. M. Haddleton, Polymeric dibromomaleimides as extremely efficient disulfide bridging bioconjugation and pegylation agents., J. Am. Chem. Soc., 2012, 134, 18471852.

28 F. F. Schumacher, M. Nobles, C. P. Ryan, M. E. B. Smith, A. Tinker, S. Caddick and J. R. Baker, In situ maleimide bridging of disulfides and a new approach to protein PEGylation, Bioconjugate Chem., 2011, 22, 132-136.

29 F. F. Schumacher, V. A. Sanchania, B. Tolner, Z. V. F. Wright, C. P. Ryan, M. E. B. Smith, J. M. Ward, S. Caddick, C. W. M. Kay, G. Aeppli, K. A. Chester and J. R. Baker, Homogeneous antibody fragment conjugation by disulfide bridging introduces 'spinostics', Sci. Rep., 2013, 3, 1525.

30 S. A. Fletcher, P. K. B. Sin, M. Nobles, E. Årstad, A. Tinker and J. R. Baker, A dual optical and nuclear imaging reagent for peptide labelling via disulfide bridging, Org. Biomol. Chem., 2015, 13, 9559-9563.

31 D. A. Richards, S. A. Fletcher, M. Nobles, H. Kossen, L. Tedaldi, V. Chudasama, A. Tinker and J. R. Baker, Photochemically re-bridging disulfide bonds and the discovery of a thiomaleimide mediated photodecarboxylation of C-terminal cysteines., Org. Biomol. Chem., 2015, 14, 455-459.

32 F. Bryden, A. Maruani, H. Savoie, V. Chudasama, M. E. B. Smith, S. Caddick and R. W. Boyle, Regioselective and stoichiometrically controlled conjugation of photodynamic sensitizers to a HER2 targeting antibody fragment., Bioconjugate Chem., 2014, 25, 611617.

33 F. F. Schumacher, J. P. M. Nunes, A. Maruani, V. Chudasama, M. E. B. Smith, K. A. Chester, J. R. Baker and S. Caddick, Next generation maleimides enable the controlled assembly of antibody-drug conjugates via native disulfide bond bridging, Org. Biomol. Chem., 2014, 12, 7261-7269.

34 R. P. Lyon, T. D. Bovee, S. O. Doronina, P. J. Burke, J. H. Hunter, H. D. Neff-laford, M. Jonas, M. E. Anderson, J. R. Setter and P. D. Senter, Reducing hydrophobicity of 
homogeneous antibody drug conjugates improves pharmacokinetics and therapeutic index, Nat. Biotechnol., 2015, 33, 733-736.

35 J. P. M. Nunes, M. Morais, V. Vassileva, E. Robinson, V. S. Rajkumar, M. E. B. Smith, R. B. Pedley, S. Caddick, J. R. Baker and V. Chudasama, Functional native disulfide bridging enables delivery of a potent, stable and targeted antibody-drug conjugate (ADC), Chem. Commun., 2015, 51, 10624-10627.

36 E. Robinson, J. P. M. Nunes, V. Vassileva, A. Maruani, J. C. F. Nogueira, M. E. B. Smith, R. B. Pedley, S. Caddick, J. R. Baker and V. Chudasama, Pyridazinediones deliver potent, stable, targeted and efficacious antibody-drug conjugates (ADCs) with a controlled loading of 4 drugs per antibody, RSC Adv., 2017, 7, 9073-9077.

37 S. O. Doronina, B. A. Mendelsohn, T. D. Bovee, C. G. Cerveny, S. C. Alley, D. L. Meyer, E. Oflazoglu, B. E. Toki, R. J. Sanderson, R. F. Zabinski, A. F. Wahl and P. D. Senter, Enhanced activity of monomethylauristatin $\mathrm{F}$ through monoclonal antibody delivery: Effects of linker technology on efficacy and toxicity, Bioconjugate Chem., 2006, 17, 114124.

38 M. Morais, J. P. M. Nunes, K. Karu, N. Forte, I. Benni, M. E. B. Smith, S. Caddick, V. Chudasama and J. R. Baker, Optimisation of the dibromomaleimide (DBM) platform for native antibody conjugation by accelerated post-conjugation hydrolysis, Org. Biomol. Chem., 2017, 15, 2947-2952.

39 L. Castañeda, A. Maruani, F. F. Schumacher, E. Miranda, V. Chudasama, K. A. Chester, J. R. Baker, M. E. B. Smith and S. Caddick, Acid-cleavable thiomaleamic acid linker for homogeneous antibody-drug conjugation., Chem. Commun., 2013, 49, 8187-8189.

40 C. R. Behrens, E. H. Ha, L. L. Chinn, S. Bowers, G. Probst, M. Fitch-Bruhns, J. Monteon, A. Valdiosera, A. Bermudez, S. Liao-Chan, J. Melnick, J.-W. Theunissen, M. R. Flory, D. Houser, K. Venstrom, Z. Levashova, P. Sauer, T.-S. Migone, E. H. van der Horst, R. L. Halcomb and D. Y. Jackson, Antibody-drug conjugates (ADCs) derived from interchain cysteine cross-linking demonstrate improved homogeneity and other pharmacological properties over conventional heterogeneous ADCs, Mol. Pharm., 2015, 12, 3986-3998. 
41 A. Maruani, M. E. B. Smith, E. Miranda, K. A. Chester, V. Chudasama and S. Caddick, A plug-and-play approach to antibody-based therapeutics via a chemoselective dual click strategy, Nat. Commun., 2015, 6, 6645.

42 C. Bahou, D. A. Richards, A. Maruani, E. A. Love, F. Javaid, S. Caddick, J. R. Baker and V. Chudasama, Highly homogeneous antibody modification through optimisation of the synthesis and conjugation of functionalised dibromopyridazinediones, $\mathrm{Org}$.

Bioomolecular Chem., 2018, 16, 1359-1366.

43 M. T. W. Lee, A. Maruani, J. R. Baker, S. Caddick and V. Chudasama, Next-generation disulfide stapling: reduction and functional re-bridging all in one, Chem. Sci., 2016, 7, 799-802.

44 S. Shao, M. H. Tsai, J. Lu, T. Yu, J. Jin, D. Xiao, H. Jiang, M. Han, M. Wang and J. Wang, Site-specific and hydrophilic ADCs through disulfide-bridged linker and branched PEG, Bioorganic Med. Chem. Lett., 2018, 28, 1363-1370.

45 S. J. Gregson, L. A. Masterson, B. Wei, T. H. Pillow, S. D. Spencer, G. Kang, S. Yu, H. Raab, G. Li, G. D. L. Phillips, J. Gunzner-toste, B. S. Sa, R. Ohri, M. Darwish, K. R. Kozak, J. Cruz-chuh, A. Polson, J. A. Flygare and P. W. Howard, Pyrrolobenzodiazepine dimer antibody-drug conjugates: synthesis and evaluation of noncleavable drug-linkers, $J$. Med. Chem., 2017, 60, 9490-9507.

46 B. S. Vollmar, B. Wei, R. Ohri, J. Zhou, J. He, S. Yu, D. Leipold, E. Cosino, S. Yee, A. F. Donohue, G. Li, G. L. Phillips, K. R. Kozak, A. Kamath, K. Xu, G. Lee, G. A. Lazar and H. K. Erickson, Attachment site cysteine thiol pKa is a key driver for site-dependent stability of THIOMAB antibody-drug conjugates, Bioconjugate Chem., 2017, 28, 25382548.

47 M. T. W. Lee, A. Maruani, D. A. Richards, J. R. Baker, S. Caddick and V. Chudasama, Enabling the controlled assembly of antibody conjugates with a loading of two modules without antibody engineering, Chem. Sci., 2017, 8, 2056-2060.

48 Z. Miao, Y. Hong, T. Zhu and A. W. Chucholowski, Drug-conjugates, conjugation methods, and uses thereof, Inter. Pat. Appl., WO/2013/173391, 2013. 
49 Q.-Y. Hu and H. Imase, Methods for making conjugates from disulfide-containing proteins, Inter. Pat. Appl., WO 2014/083505, 2014.

50 N. Assem, D. J. Ferreira, D. W. Wolan and P. E. Dawson, Acetone-linked peptides: a convergent approach for peptide macrocyclization and labeling, Angew. Chem. Int. Ed., 2015, 54, 8665-8668.

51 G. Stefanetti, Q. Y. Hu, A. Usera, Z. Robinson, M. Allan, A. Singh, H. Imase, J. Cobb, H. Zhai, D. Quinn, M. Lei, A. Saul, R. Adamo, C. A. MacLennan and F. Micoli, Sugarprotein connectivity impacts on the immunogenicity of site-selective salmonella O-antigen glycoconjugate vaccines, Angew. Chem. Int. Ed., 2015, 54, 13198-13203.

52 O. Koniev, I. Dovgan, B. Renoux, A. Ehkirch, J. Eberova, S. Cianférani, S. Kolodych, S. Papot and A. Wagner, Reduction-rebridging strategy for the preparation of ADPN-based antibody-drug conjugates, Medchemcomm, 2018, 9, 827-830.

53 N. Griebenow, A. M. Dilmaç, S. Greven and S. Bräse, Site-specific conjugation of peptides and proteins via rebridging of disulfide bonds using the thiol-yne coupling reaction, Bioconjugate Chem., 2016, 27, 911-917.

54 N. Martínez-Sáez, S. Sun, D. Oldrini, P. Sormanni, O. Boutureira, F. Carboni, I. Compañón, M. J. Deery, M. Vendruscolo, F. Corzana, R. Adamo and G. J. L. Bernardes, Oxetane grafts installed site-selectively on native disulfides to enhance protein stability and activity in vivo, Angew. Chem. Int. Ed., 2017, 56, 14963-14967.

55 A. M. Spokoyny, Y. Zou, J. J. Ling, H. Yu, Y. S. Lin and B. L. Pentelute, A perfluoroarylcysteine SNAr chemistry approach to unprotected peptide stapling, J. Am. Chem. Soc., 2013, 135, 5946-5949.

56 S. L. Kuan, T. Wang and T. Weil, Site-selective disulfide modification of proteins: expanding diversity beyond the proteome, Chem. Eur. J., 2016, 22, 17112-17129. 